\title{
Gellan gum-based hydrogels for intervertebral disc tissue-engineering applications
}

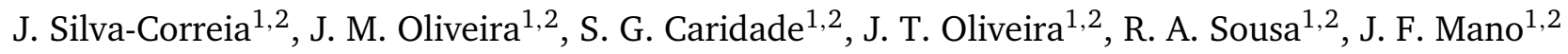 \\ and R. L. Reis ${ }^{1,2 *}$ \\ ${ }^{1}$ 3Bs Research Group - Biomaterials, Biodegradables and Biomimetics, University of Minho, Headquarters of the European Institute of \\ Excellence on Tissue Engineering and Regenerative Medicine, AvePark, S. Cláudio do Barco, 4806-909 Taipas, Guimarães, Portugal \\ ${ }^{2}$ Institute for Biotechnology and Bioengineering (IBB), PT Government-Associated Laboratory, Guimarães, Portugal
}

\begin{abstract}
Intervertebral disc (IVD) degeneration is a challenging clinical problem that urgently demands viable nucleus pulposus (NP) implant materials. The best suited biomaterial for NP regeneration has yet to be identified, but it is believed that biodegradable hydrogel-based materials are promising candidates. In this work, we have developed ionic- and photo-crosslinked methacrylated gellan gum (GG-MA) hydrogels to be used in acellular and cellular tissue-engineering strategies for the regeneration of IVDs. The physicochemical properties of the developed hydrogels were investigated by Fourier-transform infrared spectroscopy, ${ }^{1} \mathrm{H}$ nuclear magnetic resonance and differential scanning calorimetry. The swelling ability and degradation rate of hydrogels were also analysed in phosphate-buffered saline solution at physiological $\mathrm{pH}$ for a period of $\mathbf{3 0}$ days. Additionally, the morphology and mechanical properties of the hydrogels were assessed under a scanning electron microscope and dynamic compression, respectively. An in vitro study was carried out to screen possible cytotoxicity of the gellan gum-based hydrogels by culturing rat lung fibroblasts (L929 cells) with hydrogel leachables up to 7 days. The results demonstrated that gellan gum was successfully methacrylated. We observed that the produced GG-MA hydrogels possess improved mechanical properties and lower water uptake ability and degradation rate as compared to gellan gum. This work also revealed that GG-MA hydrogels are non-cytotoxic in vitro, thus being promising biomaterials to be used in IVD tissue-engineering strategies. Copyright $\odot 2010 \mathrm{John}$ Wiley \& Sons, Ltd.
\end{abstract}

Received 21 July 2010; Accepted 29 July 2010

Keywords gellan gum; hydrogels; intervertebral disc; methacrylation; nucleus pulposus; photo-crosslinking; tissue engineering

\section{Introduction}

Lower back pain (LBP) is one of the most frequently reported age- and work-related disorders (Diamond and Borenstein, 2006) and is associated with sick leave and long-term time out of employment, thus presenting a huge socio-economic impact in industrialized societies. Several

\footnotetext{
*Correspondence to: R. L. Reis, 3Bs Research GroupBiomaterials, Biodegradables and Biomimetics, University of Minho, Headquarters of the European Institute of Excellence on Tissue Engineering and Regenerative Medicine, AvePark, S. Cláudio do Barco, 4806-909 Taipas, Guimarães, Portugal. E-mail:rgreis@dep.uminho.pt
}

factors can cause LBP, but degeneration of intervertebral disc (IVD) seems to be strongly connected to the majority of cases (Urban and Roberts, 2003; Cheung and Al Ghazi, 2008). The IVD is a specialized cartilaginous structure that provides flexibility to the spine and allows limited movements while supporting compressive loads arising from body weight and muscle tension (Urban et al., 2000; Roberts et al., 2006). The avascular and relatively acellular environment of the IVDs confers limited healing potential. The stage of IVD degeneration will determine the type of therapy best suited to restore this tissue. In this respect, current treatments only attempt to treat the symptoms of pain or remove the source of pain itself, rather than addressing the regeneration of IVD tissue (Kalson 
et al., 2008). Thus, the conservative therapies (exercise, medications or physical/behaviour therapies) are used to control pain, inflammation or sleep disturbance and to improve core stability, muscle strength and motion. However, severe degeneration demands surgical treatments, which include discectomy, spinal fusion and IVD substitution. In addition to the possibility of inducing degenerative changes in adjacent discs, these treatments are not always successful and can result in limited flexibility, thus being relatively ineffective in the long term.

The regeneration of the damaged IVD using tissueengineering strategies, i.e. combining cells and scaffolds, appears to be a particularly promising alternative to the current ineffective treatments. Although a rapid increase in the understanding of the molecular and biological basis of both IVD development and degeneration has recently occurred, we now face other challenges, particularly the selection of an optimal scaffold material that enables an efficient regeneration of degenerated IVD (Richardson et al., 2007). It is obvious that the different IVD histological compartments, i.e. the nucleus pulposus (NP) and annulus fibrosus (AF), require different scaffolds. For NP replacement, injectable hydrogels derived from natural sources are ideal as these: (a) are able to mimic the native IVD environment by swelling and retaining large amounts of water (resembling ECM); (b) have the ability to withstand loads and allows the encapsulated cells to retain/induce the differentiated phenotype; and (c) allow implantation of the acellular or cell-loaded scaffold using minimally invasive procedures (e.g. injection) (Lee and Mooney, 2001; Peppas et al., 2006). An injectable hydrogel must also present a sol-gel transition mechanism suitable for clinical purposes, which means that it must have a certain viscosity and be easily crosslinked in situ to allow cell encapsulation and facilitate homogeneous cell distribution. Although a number of hydrogel systems based in natural polymers or its derivatives (e.g. chitosan, hyaluronic acid, alginate and carboxymethylcellulose) have been developed to tackle disc regeneration, they still present several problems, such as variability of production, mechanical properties and degradation rates (Roughley et al., 2006; Chou et al., 2009; Erickson et al., 2009; Jeon et al., 2009; Reza and Nicoll, 2010).

Bearing in mind these premises, we envision the use of a gellan gum-based injectable hydrogel system for the aforementioned applications. Gellan gum (GG) is an extracellular microbial anionic heteropolysaccharide consisting of glucose-glucuronic acid-glucose-rhamnose as a repeating unit and that forms a gel in the presence of metallic ions (Kang et al., 1982; Jansson et al., 1983). It is commercially available in two forms, acetylated and deacetylated, both forming thermo-reversible gels with different mechanical properties in the presence of metallic ions and upon temperature decrease. It is acid- and heatresistant and gelation occurs without the need of harsh reagents. Previously, our group (Oliveira et al., 2009a, 2010a, 2010b) demonstrated that GG hydrogels adequately support the growth and ECM deposition of human articular chondrocytes in vitro and in vivo. GG thermosensitive behaviour is suitable for injectable formulations, since gelation can be performed in situ at a temperature close to body temperature. However, as verified for other ionic-crosslinked hydrogels, significant dissolution occurs in vivo and structural integrity can be lost over time. Nevertheless, GG has a free carboxylic group per repeating unit, which can be used for functionalization and enhance its bio-stability (Oliveira et al., 2009b, 2009c). The use of photo-polymerization as an alternative method for hydrogel formation with increased structural and mechanical integrity has been attracting considerable interest in the last few years (Smeds et al., 2001; Davis et al., 2003; Baroli, 2006; Amsden et al., 2007). In this technique, polymers are modified with specific functional groups (i.e. methacrylates) that undergo free radical polymerization in the presence of a photo-initiator and upon exposure to UV light. This polymerization reaction induces the formation of covalent crosslinks between functional methacrylate groups along the backbone of the polymer chains.

In the present work, we developed ionic- and photocrosslinked gellan gum-based hydrogels to be used in IVD tissue-engineering approaches. GG was methacrylated and the physicochemical properties of the developed hydrogels were investigated. The methacrylation efficiency was studied by means of Fourier-transform infrared, ${ }^{1} \mathrm{H}$ nuclear magnetic resonance $\left({ }^{1} \mathrm{H}-\mathrm{NMR}\right)$ spectroscopic methods and differential scanning calorimetry. The morphology of the freeze-dried hydrogels was investigated using a scanning electron microscope (SEM). In addition, the swelling capacity, weight loss and dynamic mechanical properties of the prepared hydrogels were assessed. Finally, the cytotoxicity of the GG, ionic- and photo-crosslinked gellan gum-methacrylated hydrogel leachables was evaluated by carrying out a cellular viability assay (MTS test) on rat lung fibroblasts (L929 cell line) cells, which were previously in contact with the different hydrogel extract fluids.

\section{Materials and methods}

\subsection{Materials}

Low-acyl gellan gum (Gelzan $\left.{ }^{\mathrm{TM}} \mathrm{CM}, \mathrm{F}_{\mathrm{W}}=1000000\right)$, glycidyl methacrylate (97\%), methyl benzoylformate (98\%) and hydoxy-4'-(2-hydroxyethoxy)-2-methylpropiophenone were purchased from Sigma-Aldrich (USA). Unless indicated otherwise, all other reagents were obtained from Sigma-Aldrich (USA), and used as received.

\subsection{Synthesis of the methacrylated gellan gum}

Methacrylated gellan gum (GG-MA) was prepared by reacting low-acyl gellan gum (GG) with glycidyl methacrylate (GMA), based on a previously described reaction mechanism (Li et al., 2003) and according to the scheme 
(A)

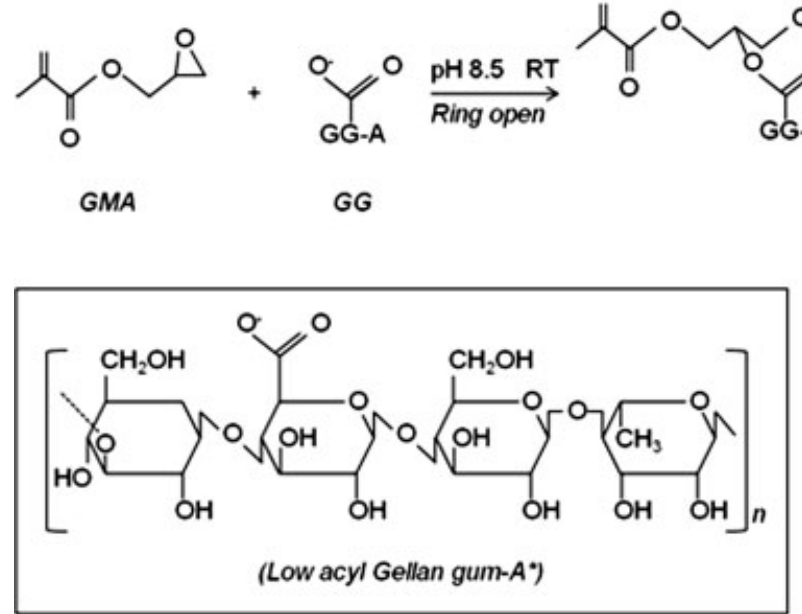

(B)
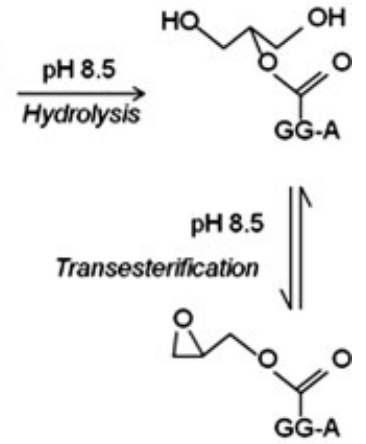

(C)

Figure 1. Schematic illustration of the pH-controlled reaction (pH 8.5) of glycidyl methacrylate (GMA) with low-acyl gellan gum (GG): (A) ring-opening product; (B) methacrylate hydrolysis mid-product; and (C) reversible trans-esterification product. ${ }^{*}$ Repeating unit of low acyl gellan gum consisting of glucose-glucuronic acid-glucose-rhamnose

presented in Figure 1. Briefly, GG was added to distilled water at room temperature under constant agitation to obtain a final concentration of $1 \% \mathrm{w} / \mathrm{v}$. The complete and homogeneous dispersion of the material was achieved after heating the solution at $90^{\circ} \mathrm{C}$. The GG solution was allowed to cool to room temperature and then an appropriate volume of GMA was added. The reaction mixture was adjusted to $\mathrm{pH} 8.5$ with $1 \mathrm{M}$ sodium hydroxide $(\mathrm{NaOH})$ and allowed to react for $24 \mathrm{~h}$ at room temperature under vigorous stirring. The $\mathrm{pH}$ was periodically adjusted to 8.5 with $1 \mathrm{M} \mathrm{NaOH}$. The reaction products were precipitated with $1 / 2$ volume of cold acetone and purified by dialysis (cellulose membrane; MW cut-off, $12 \mathrm{kDa}$ ) against distilled water for 4 days to remove residual GMA. The purified GG-MA was frozen at $-80^{\circ} \mathrm{C}$ and the powders obtained after lyophilization for 7 days.

\subsection{Preparation of the $\mathrm{GG}$, ionic- and photo-crosslinked GG - MA hydrogel discs}

In order to produce GG hydrogel discs, GG powder was dissolved in distilled water under constant agitation to obtain a $2 \% \mathrm{w} / \mathrm{v}$ solution. The mixture was then progressively heated to $90^{\circ} \mathrm{C}$ and kept at this temperature for $30 \mathrm{~min}$. Following a gradual temperature decrease to $60-65^{\circ} \mathrm{C}$, phosphate-buffered saline (PBS, $\mathrm{pH} 7.4$ ) solution was added to a final concentration of $10 \%$ $\mathrm{v} / \mathrm{v}$ in GG solution, after which the temperature was continuously decreased to $50^{\circ} \mathrm{C}$. GG discs with a diameter of $7 \mathrm{~mm}$ and height of $4 \mathrm{~mm}$ were produced by transferring the solution to a silicon mould and allowing gelation to occur at room temperature for approximately 5 min. Afterwards, the discs were equilibrated by immersion in PBS.

The ionic-crosslinked hydrogel discs were obtained from an aqueous solution of $2 \% \mathrm{w} / \mathrm{v}$ lyophilized GG-MA powders. The gel was transferred to a silicon mould and discs with a diameter of $7 \mathrm{~mm}$ and height of $4 \mathrm{~mm}$ were obtained by immersion in PBS.

The photo-crosslinked GG-MA hydrogel discs were produced by using a GG-MA solution at $2 \% \mathrm{w} / \mathrm{v}$ concentration. After complete homogenization of the solution, the photo-initiator methyl benzoylformate (MBF) was added to a final concentration of $0.1 \% \mathrm{w} / \mathrm{v}$. The gel was transferred to a silicon mould, and then hydrogel discs with a diameter of $7 \mathrm{~mm}$ and height of $4 \mathrm{~mm}$ were obtained by exposure to ultraviolet light (366 nm; UV lamp Triwood 6/36, Bresciani srl., Italy) for $10 \mathrm{~min}$. A different type of photo-initiator, hydroxy-4'-(2hydroxyethoxy)-2-methylpropiophenone (HHMPP) was alternatively used at a final concentration of $0.05 \% \mathrm{w} / \mathrm{v}$. Another type of UV light source (240-300 nm) was used to produce photo-crosslinked hydrogels in the presence of HHMPP $0.05 \% \mathrm{w} / \mathrm{v}$. The photo-crosslinked discs were further equilibrated in PBS, for $30 \mathrm{~min}$. All gellan gumbased powders were sterilized under an ethylene oxide atmosphere for the in vitro cell culture study.

\subsection{Characterization of the gellan gum-based hydrogels}

\subsubsection{Fourier-transform infrared (FTIR) spectroscopy}

The chemical modification of GG was evaluated by FTIR spectroscopy. Analysis was performed on GG as received, and using methacrylated GG-MA powder. Prior to analysis, potassium bromide $(\mathrm{KBr}$; Riedel-de Haën, Germany) was used to prepare transparent pellets $[1: 10$, sample: $\mathrm{KBr}(\mathrm{w} / \mathrm{w})]$, followed by uniaxial pressing. The infrared spectra of freeze-dried samples were recorded at room temperature on an IRPrestige-21 spectrometer (Shimadzu Corporation, Japan). All spectra were obtained at a resolution of $2 \mathrm{~cm}^{-1}$ in the range $4400-800 \mathrm{~cm}^{-1}$ for an average of 32 scans. 


\subsection{2. ${ }^{1} \mathrm{H}$-NMR spectroscopy}

The efficiency of methacrylation was also analysed by ${ }^{1} \mathrm{H}$-NMR. The ${ }^{1} \mathrm{H}$-NMR spectra of $\mathrm{GG}$ as received and GG-MA powders were recorded with a Varian Unity Plus spectrometer operating at $300 \mathrm{MHz}$, equipped with a variable temperature system. Freeze-dried samples of $10 \mathrm{mg}$ each were dissolved in $1 \mathrm{ml}$ deuterium- $d_{2}$ water $\left(\mathrm{D}_{2} \mathrm{O}\right)$ and recorded at $70^{\circ} \mathrm{C}$. The $\mathrm{D}_{2} \mathrm{O}$ peak at $4.3 \mathrm{ppm}$ was used as reference.

\subsubsection{Differential scanning calorimetry (DSC) analysis}

GG, ionic- and photo-crosslinked GG-MA hydrogel discs were produced as described above and dried in the oven at $37^{\circ} \mathrm{C}$ for 3 days. Prior to assay, samples of $10 \mathrm{mg}$ were prepared and immersed in a PBS solution for $5 \mathrm{~min}$. The DSC thermograms were obtained in a DSC Q100 apparatus (TA Instruments, USA), using a watercooling accessory and nitrogen as a purge gas (flux gas of $\approx 50 \mathrm{~cm}^{3} / \mathrm{min}$ ). Both temperature and heat flux were calibrated with indium at a scanning rate of $10^{\circ} \mathrm{C} / \mathrm{min}$. All the experiments were performed at $5^{\circ} \mathrm{C} / \mathrm{min}$, starting from $-40^{\circ} \mathrm{C}$ to $170^{\circ} \mathrm{C}$.

\subsubsection{Dynamic mechanical analysis (DMA)}

The mechanical behaviour of the $2 \% \mathrm{w} / \mathrm{v}$ GG, ionicand photo-crosslinked GG-MA hydrogel discs were characterized by DMA. The viscoelastic measurements were performed using a TRITEC Admin 8000B DMA from Triton Technology (UK), equipped with compressive mode. The measurements were carried out at $37^{\circ} \mathrm{C}$. The samples were produced using a cylindrical mould of $7 \mathrm{~mm}$ diameter and $4 \mathrm{~mm}$ height (measured accurately for each sample), and then discs were immersed in a PBS solution until the moment of the assay. The hydrogel discs were analysed while immersed in a liquid bath placed in a Teflon ${ }^{\circledR}$ reservoir. The geometry of the samples was then measured and the samples were clamped in a DMA apparatus and immersed in PBS solution. After equilibration at $37^{\circ} \mathrm{C}$, the DMA spectra were obtained during a frequency scan in the range $0.1-15 \mathrm{~Hz}$. The experiments were performed under constant strain amplitude $(50 \mu \mathrm{m})$. A small preload was applied to each sample to ensure that the entire disc surface was in contact with the compression plates before testing and the distance between plates was equal for all scaffolds being tested. Five samples were used for each condition $(n=5)$.

\subsubsection{Scanning electron microscopy (SEM) analysis}

After freeze-drying the gellan gum-based hydrogel discs, morphology of each scaffold was examined using a scanning electron microscope (Nova NanoSEM 200, FEI, USA) with an attached energy dispersive spectrometer
(EDS, Pegasus X4M). All specimens were coated with gold using a Quorum/Polaron E 6700 coater prior to analysis.

\subsubsection{In vitro degradation and water uptake studies}

Hydrogel discs were prepared according to the procedure described in Section 2.3 and lyophilized afterwards. The freeze-dried hydrogel samples $(n=3)$ were weighed (initial dry weight, $W_{d i}$ ), transferred to $15 \mathrm{ml}$ Falcon tubes and soaked in $10 \mathrm{ml} \mathrm{PBS}$ at $37^{\circ} \mathrm{C}$ under constant agitation $(60 \mathrm{rpm})$. At predetermined time points, the samples were removed, and weighed (wet weight, $W_{w}$ ). The discs were then frozen at $-80^{\circ} \mathrm{C}$, lyophilized and weighed again to determine the final dry weight $\left(\mathrm{W}_{d f}\right)$. The weight loss $\left(W_{\mathrm{L}}\right)$ and water uptake $\left(W_{\mathrm{U}}\right)$ of the discs were determined according to equations 1 and 2, respectively:

$$
\begin{aligned}
& W_{\mathrm{L}}(\%)=100 \times\left(W_{d i}-W_{d f}\right) / W_{d i} \\
& W_{\mathrm{U}}(\%)=100 \times\left(W_{w}-W_{d i}\right) / W_{d i}
\end{aligned}
$$

\subsection{In vitro screening of cytotoxicity of the gellan gum-based hydrogels}

A 3-(4,5-dimethylthiazol-2-yl)-5(3-carboxymethoxyphenyl)-2(4-sulphofenyl)-2H-tetrazolium (MTS) viability test was employed to screen the potential cytotoxicity of the GG-MA hydrogels, in accordance with ISO/EN 10993 (1992) Part 5 guidelines. The cytotoxicity of the GG as well as the ionic- and photo-crosslinked hydrogel discs prepared from GG-MA was assessed using an immortalized rat lung fibroblasts cell line (L929) purchased from the European Collection of Cell Cultures (ECACC, UK). L929 cells were grown as monolayers in Dulbecco's modified Eagle's medium (DMEM; Sigma, St. Louis, MO, USA) supplemented with $10 \%$ fetal bovine serum (FBS; Biochrom, Berlin, Germany) and $1 \%$ of an antibiotic-antimycotic mixture (Invitrogen, Carlsbad, CA, USA) containing $10000 \mathrm{U} / \mathrm{ml}$ penicillin G sodium, $10000 \mu \mathrm{g} / \mathrm{ml}$ streptomycin sulphate and $25 \mu \mathrm{g} / \mathrm{ml}$ amphotericin B as Fungizone ${ }^{\circledR}$ antimycotic in $0.85 \%$ saline. The $\mathrm{L} 929$ cells were incubated at $37^{\circ} \mathrm{C}$ in a humidified atmosphere with $5 \% \mathrm{CO}_{2}$, and the medium changed every 2 days. MTS assay was employed to assess the possible effect on cellular metabolism of leachables released from the materials within a $24 \mathrm{~h}$ extraction period. This assay is based on the bioreduction of the substrate MTS into a brown formazan product by dehydrogenase enzymes in metabolically active cells, being widely used for evaluation of cell viability. GG, ionic- and both photo-crosslinked GG-MA hydrogel discs with $0.1 \% \mathrm{w} / \mathrm{v} \mathrm{MBF}$ or $0.05 \% \mathrm{w} / \mathrm{v}$ HHMPP were prepared at a final concentration of $2 \% \mathrm{w} / \mathrm{v}$ under sterile conditions. A volume of $125 \mu \mathrm{l}$ of the different gels were transferred to each well of a 48-well tissue-culture polystyrene (TCPS) 

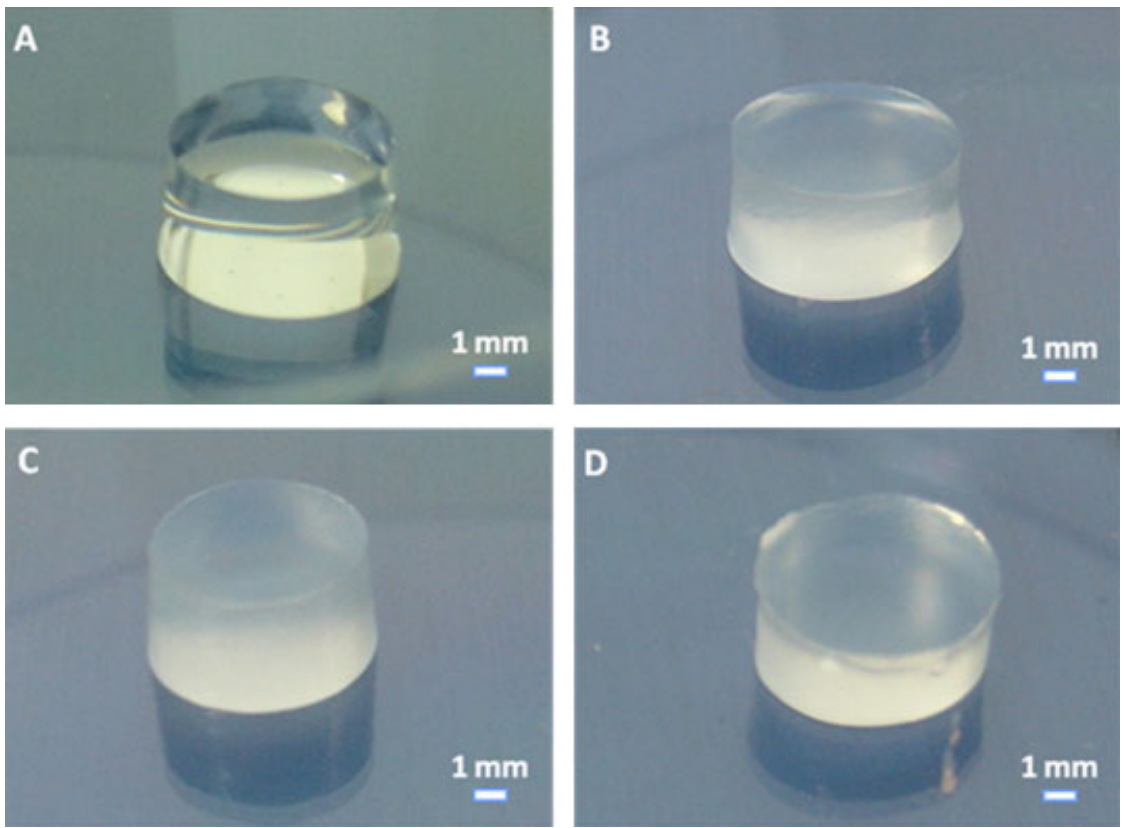

Figure 2. Images of the gellan gum-based hydrogel discs: (A) GG discs; (B) ionic-crosslinked GG-MA discs; (C) GG-MA discs photo-crosslinked with MBF $0.1 \% \mathrm{w} / \mathrm{v}$ at $366 \mathrm{~nm}$; and (D) GG-MA discs photo-crosslinked with HHMPP 0.05\% w/v at 240-300 nm

plates. The extracts fluids were prepared by introducing a minimum of 41 discs with a diameter of $15 \mathrm{~mm}$ in $50 \mathrm{ml}$ tubes containing $20 \mathrm{ml}$ complete DMEM culture medium. The tubes were incubated in a thermostatic bath at $37^{\circ} \mathrm{C}$ and $60 \mathrm{rpm}$ for $24 \mathrm{~h}$. Confluent L929 cells were detached from the culture flasks using trypsin (0.25\% trypsin-EDTA solution; Sigma) and a diluted cell suspension was prepared. L929 cells were seeded in each well of a 96-well TCPS plate (six replicates per sample) at a cell density of $2 \times 10^{4}$ cells/well. Afterwards, the cells were incubated for $24 \mathrm{~h}$ at $37^{\circ} \mathrm{C}$ in an atmosphere with $5 \% \mathrm{CO}_{2}$ to achieve $80-90 \%$ of confluence. The culture medium in each well was removed and replaced by an identical volume $(200 \mu \mathrm{l})$ of the extraction fluids. After 1,3 and 7 days, the extracts were removed and $300 \mu \mathrm{l}$ of a mixture containing serum-free culture medium without phenol red and MTS (CellTiter 96 One Solution Cell Proliferation Assay Kit; Promega, Madison, WI, USA) was added to each well. After incubation for $3 \mathrm{~h}$ at $37{ }^{\circ} \mathrm{C}$ and with $5 \% \mathrm{CO}_{2}$, the optical density (OD) was measured at $490 \mathrm{~nm}$ using a plate reader (Molecular Devices, SunnyVale, CA, USA). A latex rubber extract was used as positive control for cell death and culture medium was used as a negative control representing the ideal situation for cell proliferation. The percentage of cell viability was calculated after normalization with the mean OD value obtained for the negative control. The MTS assay was repeated three times $(n=18)$.

\subsection{Statistical analysis}

Statistical analysis (GraphPad Prism; GraphPad Software, San Diego, CA, USA) was performed using one-way analysis of variance followed by Bonferroni post-test, and significance was set at $p<0.05$.

\section{Results and discussion}

\subsection{Physicochemical characterization of the developed gellan gum-based hydrogels}

Although gellan gum (GG) hydrogels presents many advantages for finding application in tissue-engineering scaffolding, there are still some features which need to be tailored in order to be more adequate for in vivo use. Some aspects that should be addressed are related, on one hand, to the need to optimize their gelation conditions (e.g. time and temperature) in order to be clinically suitable and, on the other hand, the mechanical performance and in vitro stability should be also adjusted to mimic the functional properties of the tissue aimed to regenerate. To improve the in vitro stability of GG hydrogels while envisioning obtaining a photo-crosslinkable polymer, we thought to chemically modify this gel-forming polysaccharide with glycidyl methacrylate (GMA) (Figure 1). In this study, we have developed different gellan gum-based hydrogels for tissue engineering and regenerative medicine applications (Figure 2). These innovative hydrogels were obtained either by ionic (in the presence of monovalent cations) or photo-crosslinking (in the presence of two different types of free radical initiators, MBF and HHMPP) of modified low-acyl GG. As otherwise mentioned, low acyl GG is capable of physical gelation in the presence of ions and upon temperature decrease (Miyoshi et al., 1996). Chemical modification of GG by methacrylation of the free carboxylic groups enables enhancement of their mechanical properties by allowing a stronger crosslinking of hydrogel network.

The methacrylation efficiency was studied by Fouriertransform infrared (FTIR), ${ }^{1} \mathrm{H}$-NMR spectroscopic methods and differential scanning calorimetry (DSC) analysis. 


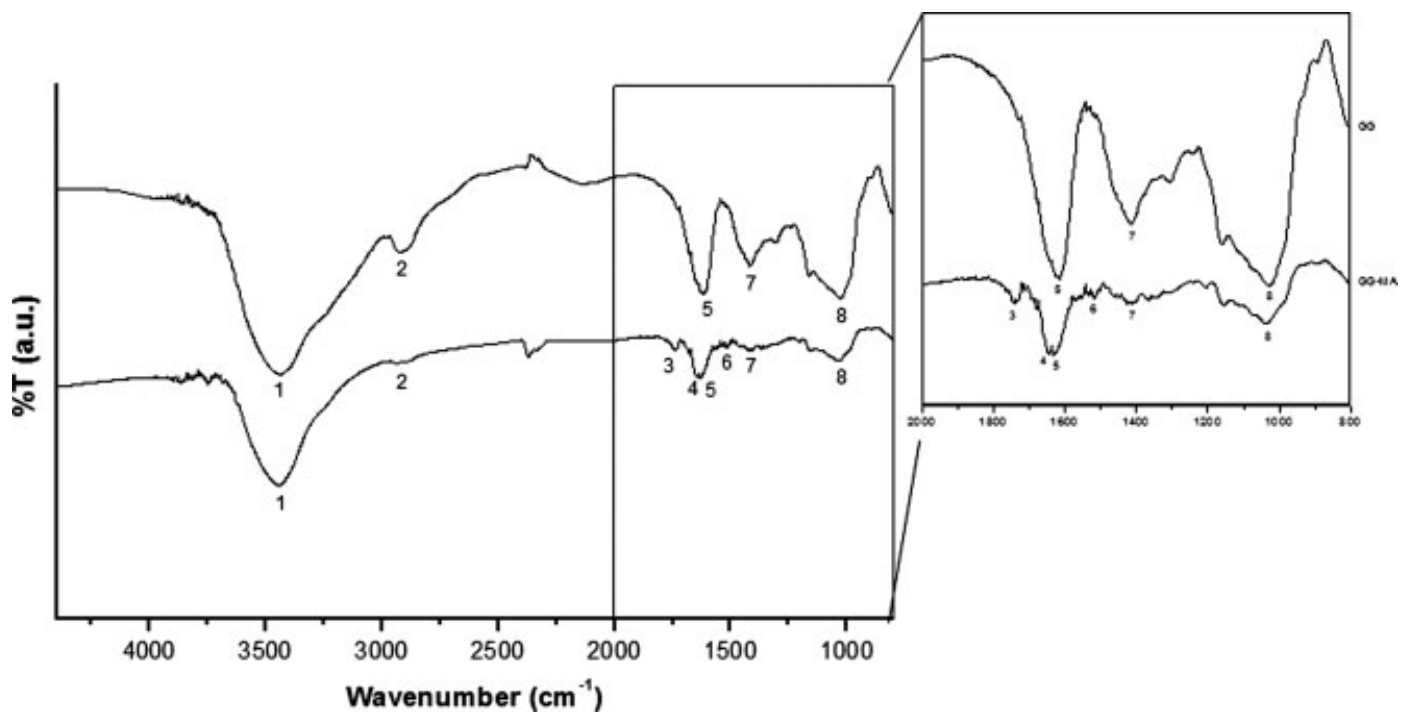

Figure 3. FTIR spectra of low-acyl gellan gum (GG) and methacrylated gellan gum (GG-MA) powders. The absorption band around $1645 \mathrm{~cm}^{-1}$, typical of the $\mathrm{C}=\mathrm{C}$ bond of glycidyl methacrylate, is present in GG-MA. The appearance of a peak at $1738 \mathrm{~cm}^{-1}$ assigned to the $\mathrm{C}=\mathrm{O}$ bond of the ester is also indicative of its introduction in the gellan gum chain. Peaks assignment can be seen in Table 1

The synthesis of the methacrylated gellan gum (GG-MA) is evidenced in Figure 3. Typical GG absorption bands were present in both GG and GG-MA FTIR spectra at $3420,2920,1618,1412$ and $1037 \mathrm{~cm}^{-1}$ (peak assignment in Table 1), as described by Sudhamani et al. (2003). The FTIR spectra of GG-MA revealed the appearance of new peaks at 1738 and $1645 \mathrm{~cm}^{-1}$ which are typical of carbonyl stretching vibration of an ester and double bond $(v \mathrm{C}=\mathrm{C})$ stretches, respectively (Table 1 ). These results indicate that, according to the reaction scheme presented in Figure 1, the ring-opening product (A) was successfully obtained from the reaction between GG and GMA. Gellan gum has hydroxyl and carboxyl groups in its structure that could undergo a nucleophilic substitution reaction with GMA. As described for the methacrylation of chondroitin sulfate by Li et al. (2003), under controlled basic conditions $(\mathrm{pH}$ 8.5) the most favourable reaction would be the carboxyl groups. Accordingly, the absorption band at $1412 \mathrm{~cm}^{-1}$ corresponding to the symmetric carboxyl stretching of GG is almost imperceptible in the methacrylated material (GG-MA). The insertion of methacrylate groups in GG structure was corroborated by ${ }^{1} \mathrm{H}$-NMR analysis (Figure 4). The chemical shift for unmodified GG presented characteristic signals at 5.15 and $1.32 \mathrm{ppm}$, corresponding to H-1 and H-6 of the $\alpha$-anomers of L-rhamnopyranosyl residue, as described by Tako et al. (2009). Additionally, the signals at $4.73 \mathrm{ppm}$ and $4.55 \mathrm{ppm}$ should be attributed to D-glucopyranosyl and D-glucuropyranosyl residues, respectively. These characteristic signals were also present in the chemical shift for GG-MA. The GG-MA spectra also showed the appearance of singlets at 1.96, 5.77 and $6.18 \mathrm{ppm}$ (black arrows), which are ascribed to the protons newly formed from the reaction of GG with GMA. These data clearly revealed that methacrylation was successfully achieved. As observed for other described
Table 1. Peak assignment of the absorption bands identified in FTIR spectra of GG and GG-MA powders

\begin{tabular}{|c|c|c|}
\hline \multicolumn{2}{|c|}{ Peak $\left(\mathrm{cm}^{-1}\right)$} & Assignment \\
\hline 1 & 3420 & $\mathrm{O}-\mathrm{H}$ stretching peak \\
\hline 2 & 2920 & $\mathrm{C}-\mathrm{H}$ strech \\
\hline 3 & 1738 & $\begin{array}{l}\text { Typical }(v C=0) \text { carbonyl stretching vibration of an } \\
\text { ester }\end{array}$ \\
\hline 4 & 1645 & $\begin{array}{l}\text { Typical }(v C=C) \text { stretches; at } 1636 \text { is not well } \\
\text { discernible due to overlapping with the asymmetric } \\
C 00^{-} \text {stretching band }\end{array}$ \\
\hline 5 & 1618 & Asymmetric $\mathrm{COO}^{-}$stretching \\
\hline 6 & 1536 & C-C stretching \\
\hline 7 & 1412 & Symmetric $\mathrm{COO}^{-}$stretching \\
\hline 8 & 1037 & $\mathrm{C}-\mathrm{O}$ stretching \\
\hline
\end{tabular}

methacrylation reactions (Li et al., 2003; Amsden et al., 2007; Jeon et al., 2009), the insertion of methacrylate groups is identified in ${ }^{1} \mathrm{H}$-NMR spectra by the appearance of a peak due to the methyl proton of methacrylate (1.96 ppm) and two peaks from vinyl-proton peaks (5.77 and $6.18 \mathrm{ppm}$ ).

Figure 5 displays the DSC profiles of dried discs made of GG, ionic-crosslinked GG-MA, GG-MA photo-crosslinked with $0.1 \% \mathrm{MBF}$ and GG-MA photo-crosslinked with $0.05 \%$ HHMPP. The appearance of two or more endothermic peaks in the heating DSC curves of GG-MA may be an indication of the presence of junction zones with different bonding energies or different rotational freedoms (Figure 5A) (Nishinari, 1997). Higher temperature endothermic peaks, as observed for GG-MA in comparison to GG, could be attributed to the melting of the zones with higher bonding energies or with lower rotational freedoms. Figures 5B, C also show the DSC profile of photo-crosslinked discs produced in the presence of the photo-initiators MBF and HHMPP. Once more, the appearance of multiple endothermic peaks in the heating DSC curves of photo-crosslinked GG-MA 


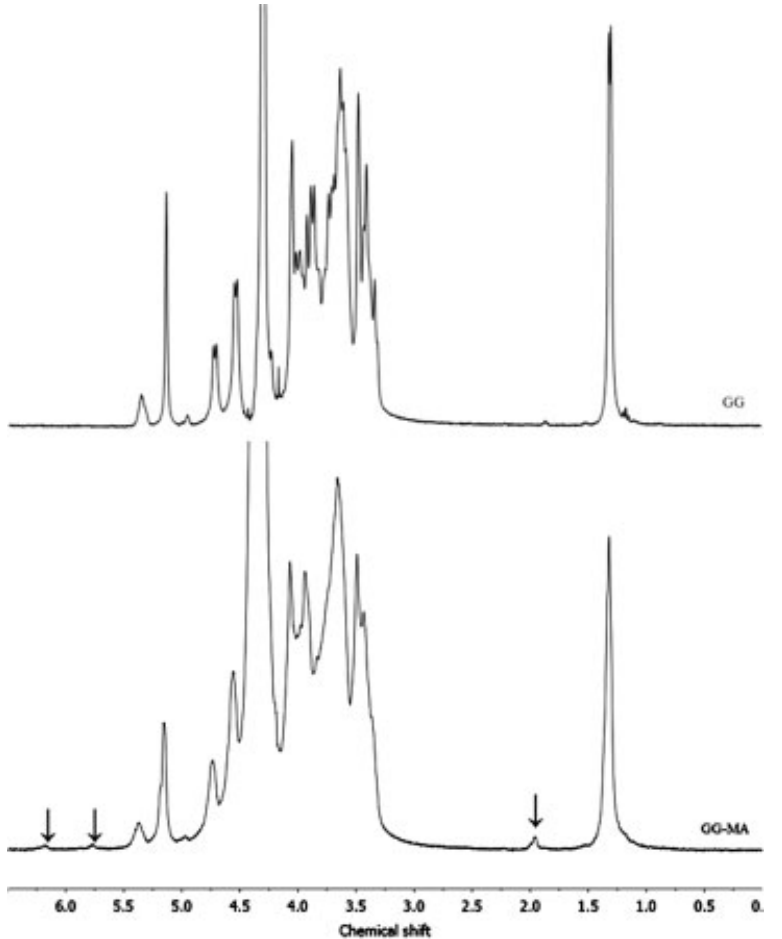

Figure 4. ${ }^{1} \mathrm{H}-\mathrm{NMR}$ spectra of gellan gum (GG) and methacrylated gellan gum (GG-MA) performed in $\mathrm{D}_{2} \mathrm{O}$ at $70^{\circ} \mathrm{C}$

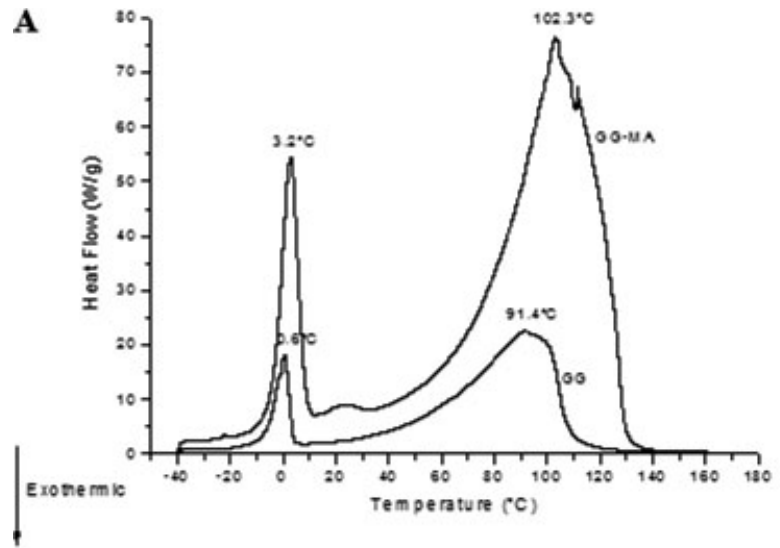

hydrogel discs suggests the existence of connections with different thermal stabilities. The DSC results revealed that GG-MA and photo-crosslinked GG-MA form ordered structures involving more thermally stable junction zones.

Mechanical properties, morphology, swelling capacity and weight loss of the prepared gellan gum-based hydrogels were also investigated. Figure 6 shows the dynamic mechanical analysis (DMA) of gellan gumbased hydrogel discs measured in wet state (PBS) and using a Teflon ${ }^{\circledR}$ reservoir, throughout a physiological relevant frequency range $(0.1-15 \mathrm{~Hz})$. The mechanical behaviour of GG discs were compared to ionic- and photocrosslinked GG-MA discs with either $0.1 \%$ MBF or $0.05 \%$ HHMPP. The storage modulus of the hydrogels tends to increase with increasing frequency but behaves very like a linear viscoelastic material (Figure 6A). This means that the tested hydrogels are elastic in a certain extent. Although intact human intervertebral discs exhibit linear viscoelasticity during compression (Holmes and Hukins, 1996), this slight increase in storage modulus has already been reported in a sheep model for frequencies in the range $0.1-10 \mathrm{~Hz}$ (Leahy and Hukins, 2001). The storage modulus at $1 \mathrm{~Hz}$ of photo-crosslinked GG-MA discs with $\operatorname{MBF}(122.8 \pm 8.3 \mathrm{kPa})$ and HHMPP $(151.2 \pm 29.9 \mathrm{kPa})$ is higher than that of GG hydrogels $(56.2 \pm 1.4 \mathrm{kPa})$, which can be attributed to the more compact microstructure,
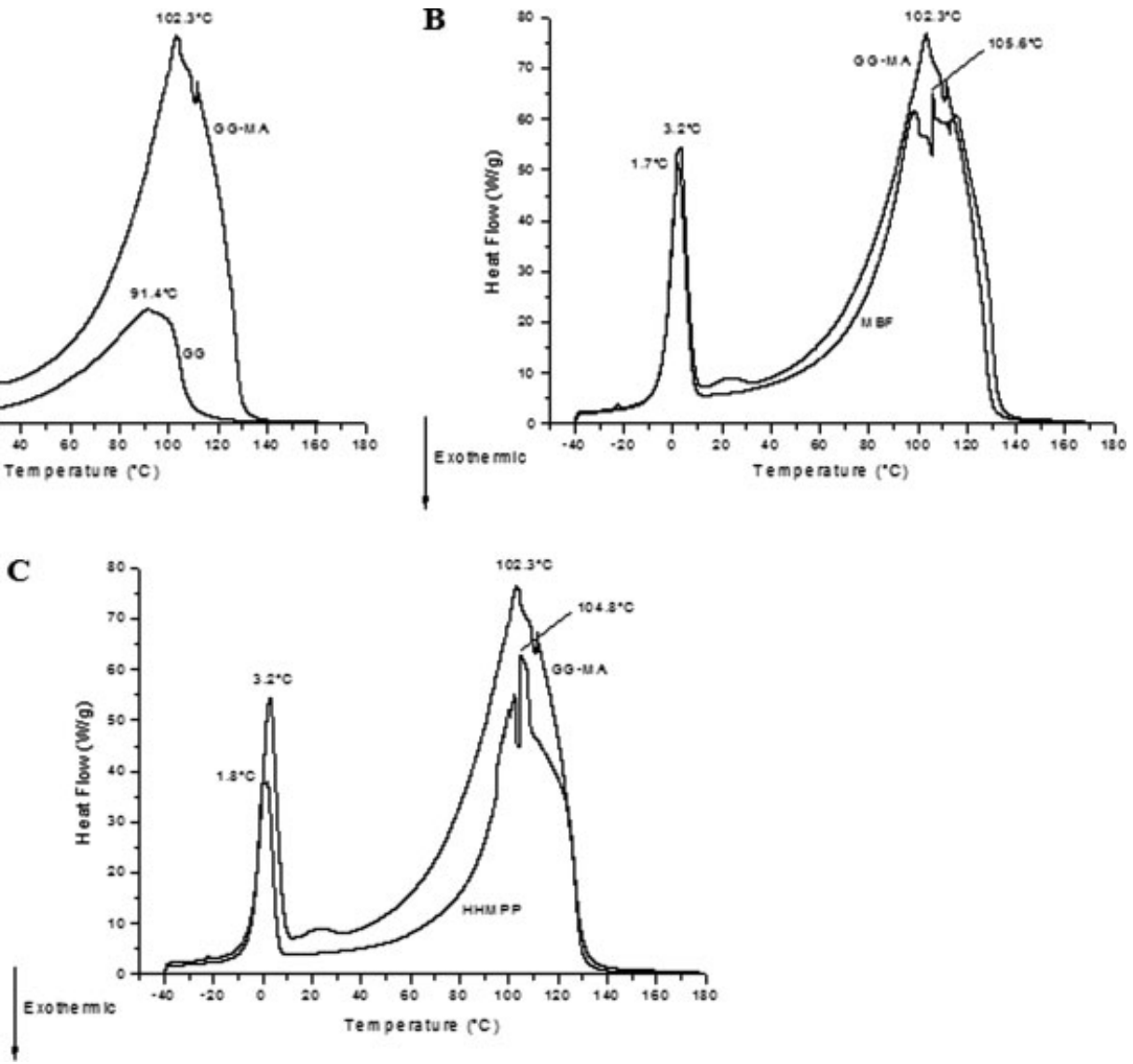

Figure 5. Heating DSC curves of: (A) gellan gum (GG) and ionic-crosslinked GG-MA discs (GG-MA); (B) GG-MA discs photo-crosslinked with MBF $0.1 \% \mathrm{w} / \mathrm{v}$ (MBF) and ionic-crosslinked GG-MA discs (GG-MA); and (C) GG-MA discs photo-crosslinked with HHMPP 0.05\% w/v (HHMPP) and ionic-crosslinked GG-MA discs (GG-MA) 

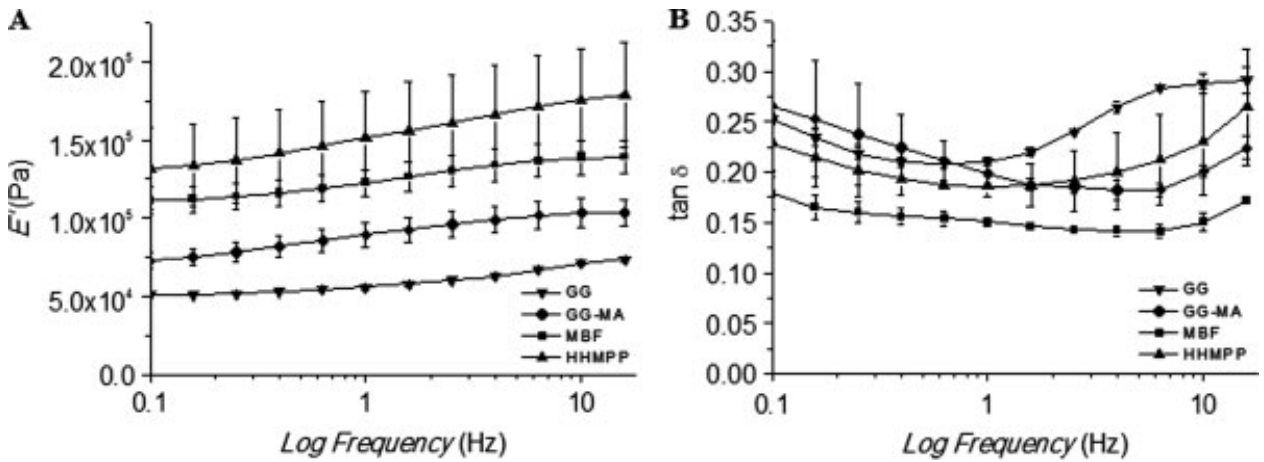

Figure 6. DMA analysis of gellan gum-based hydrogel discs: (A) the storage $\left(E^{\prime}\right)$ modulus; (B) loss factor (tan $\delta$ ) measured in PBS at $37^{\circ} \mathrm{C}$. Gellan gum discs (GG) ionic-crosslinked GG-MA discs (GG-MA), GG-MA discs photo-crosslinked with MBF $0.1 \%$ w/v at $366 \mathrm{~nm}$ (MBF) and GG-MA discs photo-crosslinked with HHMPP $0.05 \% \mathrm{w} / \mathrm{v}$ at 240-300 nm (HHMPP)
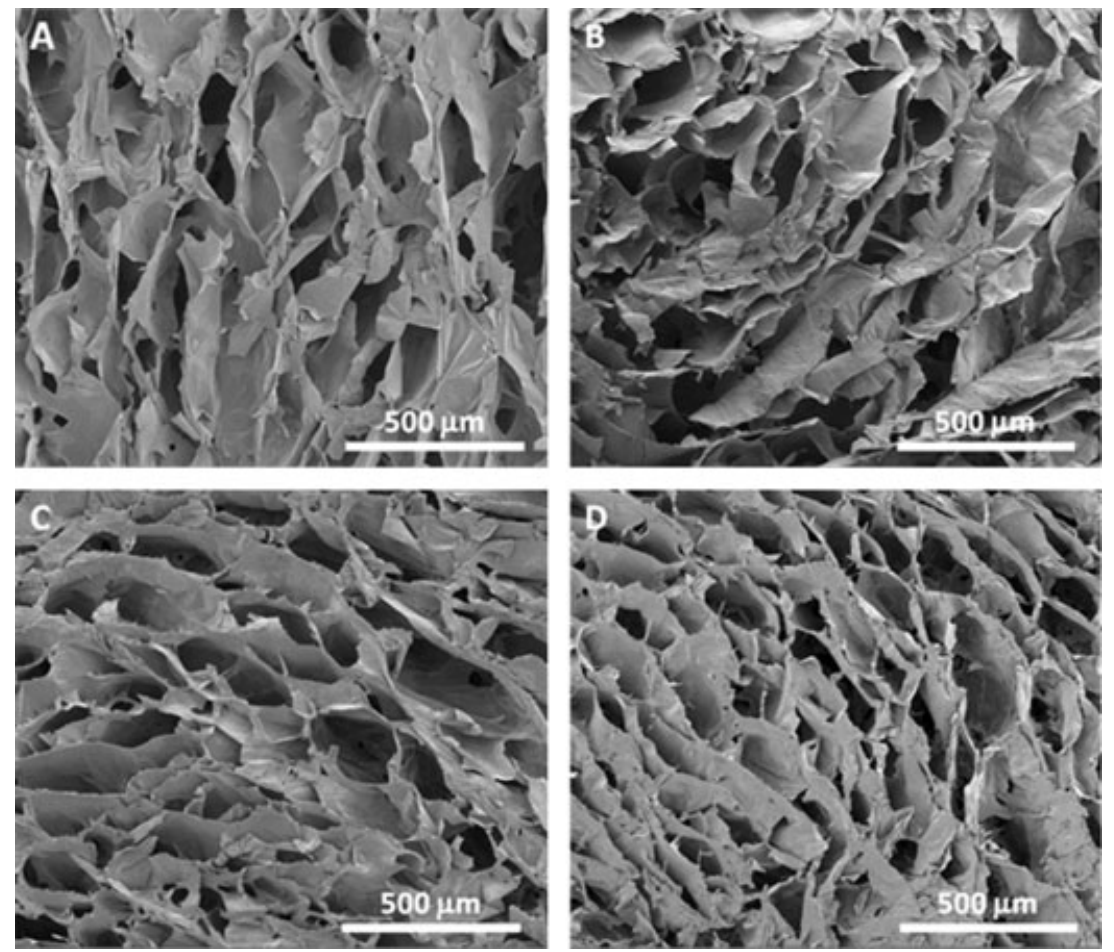

Figure 7. SEM images of the cross-section of freeze-dried gellan gum-based hydrogel discs prepared for the in vitro studies: (A) GG discs; (B) ionic-crosslinked GG-MA discs; (C) GG-MA discs photo-crosslinked with MBF $0.1 \% \mathrm{w} / \mathrm{v}$ at $366 \mathrm{~nm}$; and (D) GG-MA discs photo-crosslinked with HHMPP $0.05 \% \mathrm{w} / \mathrm{v}$ at $240-300 \mathrm{~nm}$

i.e. higher crosslinking density. The higher crosslinking degree, the higher elasticity will present the hydrogels discs. Ionic-crosslinked GG-MA hydrogels presented an intermediate behaviour $(89.5 \pm 7.4 \mathrm{kPa})$. Loss factor (tan $\delta$ ) for GG and ionic- and photo-crosslinked GG-MA discs is found in Figure 6B. Values varied between 0.15 and 0.21 at $1 \mathrm{~Hz}$, with GG displaying the higher value. Differences in water retention and microstructure compaction of the hydrogels may explain these observations. Iatridis et al. (1997a) reported a shear modulus of 7-20 kPa measured by rheology for the native human nucleus pulposus (NP). From this result, a Young's modulus of 20-60 kPa can be inferred (Leahy and Hukins, 2001), which is close to the results obtained for GG hydrogels. However, the results from human tissue were obtained from post mortem evaluation, which could influence the properties of the NP (Iatridis et al., 1997b). In a sheep model, Leahy and Hukins (2001) determined by dynamic compression that NP tissue has a storage modulus of $64 \pm 28 \mathrm{kPa}$ and a loss factor of $0.33 \pm 0.07$. The NP of sheep is comparable to the human NP: it has a similar appearance and water content and thus it is believed to be a reasonable model for human intervertebral discs (Reid et al., 2002). Ionic- and photo-crosslinked GG-MA hydrogels presented a higher storage modulus than that of native human NP.

Several authors (Oliveira et al., 2006; Wu et al., 2010) have been developing highly porous scaffolds for tissue engineering by means of freezing a polymer solution and subsequently freeze-drying. Figure 7 shows the images 

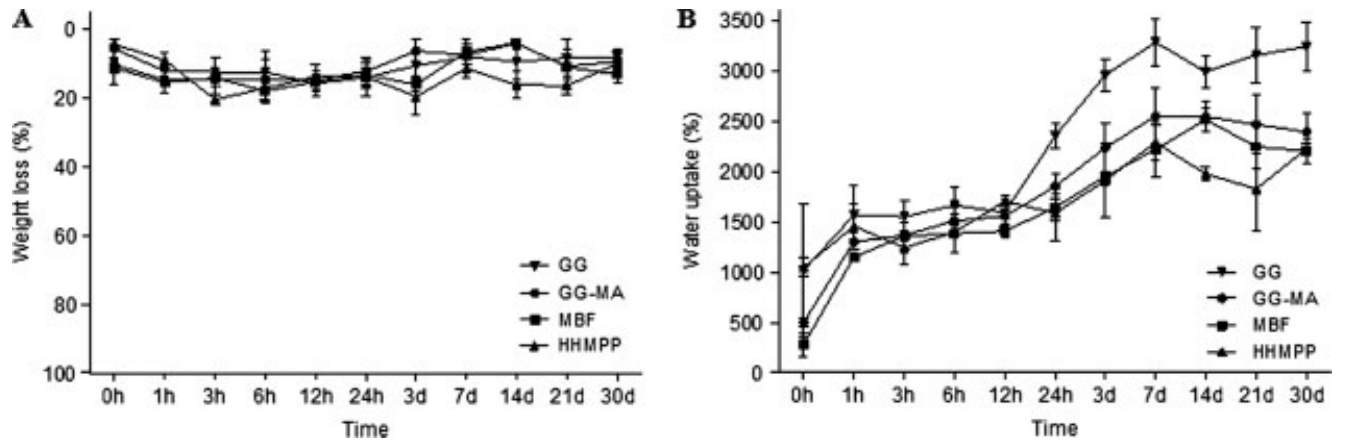

Figure 8. Weight loss (A) and water uptake (B) of: gellan gum (GG); ionic-crosslinked GG-MA hydrogel discs (GG-MA); GG-MA photo-crosslinked with photo-initiator MBF $0.1 \% \mathrm{w} / \mathrm{v}$ at $366 \mathrm{~nm}$ (MBF); and GG-MA photo-crosslinked with HHMPP $0.05 \%$ w/v at 240-300 nm (HHMPP). All samples were soaked in PBS ( $\mathrm{pH} 7.4 ; 37^{\circ} \mathrm{C}$ and $60 \mathrm{rpm}$ ) for times in the range $0-30$ days

obtained from SEM of the freeze-dried gellan gumbased hydrogels used in the in vitro degradation and swelling studies. From images of typical transverse crosssections of the scaffolds, it is possible to observe that all samples possess pores with a typical spongy threedimensional (3D) morphology, with open macropores and anisotropic porosity. No differences were found in the morphology of the interior of all gellan gumbased hydrogels analysed, although differences in the crosslinking degree are expected.

Figure 8 shows the weight loss and water uptake ability of the developed gellan gum-based hydrogels soaked in PBS at $37^{\circ} \mathrm{C}$ and $60 \mathrm{rpm}$. These results showed that no significant degradation was observed for all the freeze-dried gellan gum-based hydrogel discs tested (i.e. GG, ionic- and photo-crosslinked GG-MA with MBF and HHMPP) after 30 days (Figure 8A). Regarding the swelling ability of the hydrogels, the values for water uptake in all the hydrogel discs obtained from GG-MA (i.e. ionic- and photo-crosslinked GG-MA with MBF and HHMPP) were lower than the values observed for GG (Figure 8B). This can be attributed to a higher crosslinking density, consistent with a tighter matrix, which thus is less able to swell.

\subsection{In vitro cell culture study}

In order to be validated as an in situ gelable biomaterial for in vivo use, a developed gellan gum-based hydrogel or its degradation products should not elicit any deleterious effect on cells functions. Cytotoxicity of the GG, ionicand photo-crosslinked GG-MA hydrogels leachables was evaluated by carrying out a cellular viability assay (MTS test) on rat lung fibroblasts (L929 cells) and following (Figure 9). These data revealed that L929 cells were metabolically active after contact with the different extract fluids for all the periods tested. No statistically significant differences were found between the materials and negative control and the tests revealed that the hydrogels were non-cytotoxic. Thus, it can be stated that the concentration of the polymer, the degree of methacrylation and the type and concentration of photoinitiator (MBF or HHMPP) do not have a deleterious effect

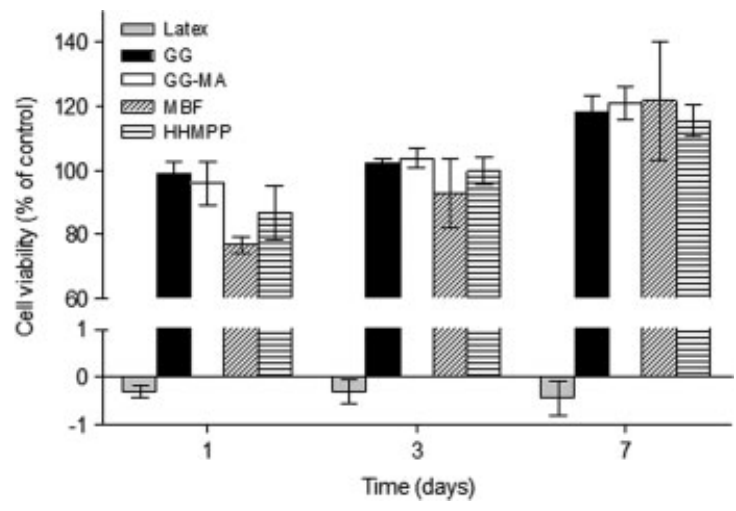

Figure 9. Cytotoxicity screening of the gellan gum-based hydrogel leachables using rat lung fibroblasts (L929 cells): gellan gum (GG); ionic-crosslinked GG-MA hydrogel discs (GG-MA); GG-MA photo-crosslinked with photo-initiator MBF $0.1 \% \mathrm{w} / \mathrm{v}$ at $366 \mathrm{~nm}$ (MBF); and GG-MA photo-crosslinked with HHMPP $0.05 \% \mathrm{w} / \mathrm{v}$ at $240-300 \mathrm{~nm}$ (HHMPP). Latex extract (Latex) and standard culture medium were used as positive and negative controls, respectively. An MTS assay was performed after 1, 3 and 7 days

on cellular metabolism. In turn, a toxic effect of latex for cell death (positive control) on cell viability was also clearly observed.

Herein, we have demonstrated that GG can be chemically modified. The structure of the developed gellan gumbased hydrogels was characterized and gelation conditions optimized. We have also demonstrated that GG-MA hydrogels are ionic- and photo-crosslinkable, present higher mechanical properties (acellular hydrogels) as compared to native NP and are non-cytotoxic. Thus, the replacement of NP compartment of the disc with these promising biomaterials, either seeded or non-seeded with cells, which can be implanted as injectable gels while gelation is possible to occur in situ could represent an alternative treatment strategy for treating IVD degeneration.

\section{Conclusions}

Chemical modification of gellan gum (GG) with glycidyl methacrylate is feasible and allows obtaining both ionic- and photo-crosslinkable methacrylated gellan gum 
(GG-MA) hydrogels. Furthermore, it was possible to optimize hydrogel gelation time within a few minutes by adjusting the concentration and type of the photo-initiator used, which is a must for minimally invasive surgeries. Photo-initiator methyl benzoylformate was added at a final concentration of $0.1 \% \mathrm{w} / \mathrm{v}$ to $2 \% \mathrm{GG}-\mathrm{MA}$ and allowed to photo-crosslink the GG-MA hydrogels by exposure to ultraviolet light $(366 \mathrm{~nm})$ within $10 \mathrm{~min}$. The results demonstrated that ionic-crosslinked GG-MA hydrogels can be produced, but photo-crosslinking improved the mechanical properties of the GG-MA hydrogels. After 30 days, the water uptake ability of GG-MA hydrogels decreased as compared to GG, while weight loss did not significantly vary. Biocompatibility studies also showed that the gellan gum-based hydrogels leachables are non-cytotoxic to L929 cells. Therefore, the proposed gellan gum-based hydrogels may be useful in intervertebral disc regeneration as acellular or cellular substitutes of the nucleus pulposus, and our future studies will be focused in these particular applications.

\section{Acknowledgements}

The authors are grateful for funds provided by the Portuguese Foundation for Science and Technology (FCT) through the POCTI and FEDER programmes, including Project ProteoLight (Grant No. PTDC/FIS/68517/2006). This work was also carried out with the support of the European Union-funded Collaborative Project Disc Regeneration (Grant No. NMP3-LA-2008-213904).

\section{References}

Amsden BG, Sukarto A, Knight DK, et al. 2007; Methacrylated glycol chitosan as a photopolymerizable biomaterial. Biomacromolecules 8: 3758-3766.

Baroli B. 2006; Photopolymerization of biomaterials: issues and potentialities in drug delivery, tissue engineering, and cell encapsulation applications. J Chem Technol Biotechnol 81: 491-499.

Cheung KMC, Al Ghazi S. 2008; Current understanding of low back pain and intervertebral disc degeneration: epidemiological perspectives and phenotypes for genetic studies. Curr Orthop 22: 237-244.

Chou AI, Akintoye SO, Nicoll SB. 2009; Photo-crosslinked alginate hydrogels support enhanced matrix accumulation by nucleus pulposus cells in vivo. Osteoarthr Cartilage 17: 1377-1384.

Davis KA, Burdick JA, Anseth KS. 2003; Photoinitiated crosslinked degradable copolymer networks for tissue engineering applications. Biomaterials 24: 2485-2495.

Diamond S, Borenstein D. 2006; Chronic low back pain in a working-age adult. Best Pract Res Clin Rheumatol 20: 707-720.

Erickson IE, Huang AH, Sengupta S, et al. 2009; Macromer density influences mesenchymal stem cell chondrogenesis and maturation in photocrosslinked hyaluronic acid hydrogels. Osteoarthr Cartilage 17: 1639-1648.

Hamcerencu M, Desbrieres J, Khoukh A, et al. 2008; Synthesis and characterization of new unsaturated esters of gellan gum. Carbohydr Polym 71: 92-100.

Holmes AD, Hukins DWL. 1996; Analysis of load-relaxation in compressed segments of lumbar spine. Med Eng Phys 18: 99-104.

Iatridis JC, Setton LA, Weidenbaum M, et al. 1997a; The viscoelastic behavior of the non-degenerate human lumbar nucleus pulposus in shear. $J$ Biomech $\mathbf{3 0}$ 1005-1013.

Iatridis JC, Setton LA, Weidenbaum M, et al. 1997b; Alterations in the mechanical behavior of the human lumbar nucleus pulposus with degeneration and aging. $J$ Orthopaed Res 15: 318-322.

ISO/EN10993-5. 1992; Biological evalua tion of medical devices - Part 5: Tests for cytotoxicity: in vitro methods. International Standards Organization: Genève, Switzerland.
Jansson P-E, Lindberg B, Sandford PA. 1983; Structural studies of gellan gum, an extracellular polysaccharide elaborated by Pseudomonas elodea. Carbohydr Res 124: 135-139.

Jeon O, Bouhadir KH, Mansour JM, et al. 2009; Photocrosslinked alginate hydrogels with tunable biodegradation rates and mechanical properties. Biomaterials $\mathbf{3 0}$ 2724-2734.

Kalson N, Richardson S, Hoyland J. 2008; Strategies for regeneration of the intervertebral disc. Regen Med $\mathbf{3}$ 717-729.

Kang KS, Veeder GT, Mirrasoul PJ, et al. 1982; Agar-like polysaccharide produced by a Pseudomonas species: production and basic properties. Appl Environ Microbiol 43: 1086-1091.

Leahy JC, Hukins DWL. 2001; Viscoelastic properties of the nucleus pulposus of the intervertebral disk in compression. J Mater Sci Mater Med 12: 689-692.

Lee KY, Mooney DJ. 2001; Hydrogels for tissue engineering. Chem Rev 101: 1869-1880.

Li Q, Wang D-A, Elisseeff JH. 2003; Heterogeneous-phase reaction of glycidyl methacrylate and chondroitin sulfate: mechanism of ring-opening transesterification competition. Macromolecules 36: 2556-2562.

Miyoshi E, Takaya T, Nishinari K. 1996; Rheological and thermal studies of gel-sol transition in gellan gum aqueous solutions. Carbohydr Polym 30: 109-119.

Nishinari K. 1997; Rheological and DSC study of sol-gel transition in aqueous dispersions of industrially important polymers and colloids. Colloid Polym Sci 275: 1093-1107.

Oliveira JM, Rodrigues MT, Silva SS, et al 2006; Novel hydroxyapatite/chitosan bilayered scaffold for osteochondral tissue-engineering applications: scaffold design and its performance when seeded with goat bone marrow stromal cells. Biomaterials 27: 6123-6137.

Oliveira JT, Gardel LS, Rada T, et al. 2010b; Injectable gellan gum hydrogels with autologous cells for the treatment of rabbit articular cartilage defects. $J$ Orthop Res [Epub ahead of print].
Oliveira JT, Martins L, Picciochi R, et al. 2009b; Gellan gum: a new biomaterial for cartilage tissue engineering applications. $J$ Biomed Mater Res A 93A: 852-863.

Oliveira JT, Santos TC, Martins L, et al. 2010a; Gellan gum injectable hydrogels for cartilage tissue engineering applications: in vitro studies and preliminary in vivo evaluation. Tissue Eng A 16: 343-353.

Oliveira JT, Santos TC, Martins L, et al. 2009a; Performance of new gellan gum hydrogels combined with human articular chondrocytes for cartilage regeneration when subcutaneously implanted in nude mice. J Tissue Eng Regen Med 3: 493-500.

Oliveira JT, Sousa RA, Reis RL. 2009c; Gellan gum-based hydrogels for regenerative medicine and tissue engineering applications, its system, and processing devices. Patent No. WO/2009/101518: Portugal.

Peppas NA, Hilt JZ, Khademhosseini A, et al. 2006; Hydrogels in biology and medicine: from molecular principles to bionanotechnology. Adv Mater 18: 1345-1360.

Reid JE, Meakin JR, Robins SP, et al. 2002; Sheep lumbar intervertebral discs as models for human discs. Clin Biomech 17: 312-314.

Reza AT, Nicoll SB. 2010; Characterization of novel photocrosslinked carboxymethylcellulose hydrogels for encapsulation of nucleus pulposus cells. Acta Biomater 6: 179-186.

Richardson SM, Mobasheri A, Freemont AJ, et al. 2007; Intervertebral disc biology, degeneration and novel tissue engineering and regenerative medicine therapies. Histol Histopathol 22: 1033-1041.

Roberts S, Evans H, Trivedi J, et al. 2006; Histology and pathology of the human intervertebral disc. $J$ Bone Joint Surg Am 88: $10-14$.

Roughley P, Hoemann C, DesRosiers E, et al. 2006; The potential of chitosan-based gels containing intervertebral disc cells for nucleus pulposus supplementation. Biomaterials 27: 388-396.

Smeds KA, Pfister-Serres A, Miki D, et al. 2001; Photocrosslinkable polysaccharides for in situ hydrogel formation. $J$ Biomed Mater Res 54: 115-121. 
Sudhamani SR, Prasad MS, Udaya Sankar K. 2003; DSC and FTIR studies on gellan and polyvinyl alcohol (PVA) blend films. Food Hydrocolloids 17: 245-250.

Tako M, Teruya T, Tamaki Y, et al. 2009; Molecular origin for rheological characteristics of native gellan gum. Colloid Polym Sci 287: 1445-1454.
Urban JPG, Roberts S. 2003; Degeneration of the intervertebral disc. Arthritis Res Ther 5: $120-130$.

Urban JPG, Roberts S, Ralphs JR. 2000; The nucleus of the intervertebral disc from development to degeneration. Am Zool 40: 53-61.
Wu X, Liu Y, Li X, et al. 2010; Preparation of aligned porous gelatin scaffolds by unidirectional freeze-drying method. Acta Biomater 6: 1167-1177. 